\title{
Sesgo cognitivo y redes neuronales artificiales aplicados en una BCI para clasificación de señales neuronales biológicas a palabras dicotómicas "SI-NO" obtenidas mediante un EEG: Speechless Talk
}

\author{
Bladimir Serna ${ }^{1}$, Rosario Baltazar ${ }^{1}$, Martha Rocha ${ }^{1}$, \\ Delia Irazú Hernández Farías ${ }^{2}$, Miguel Ángel Casillas-Araiza ${ }^{1}$, Victor Zamudio ${ }^{1}$ \\ 1 Tecnológico Nacional de México, \\ Instituto Tecnológico de León, \\ México \\ ${ }^{2}$ Universidad Politecnica de Valencia, Valencia, \\ España \\ \{bladimir.serna,marthaalicia.rocha\}@itleon.edu.mx, charobalmx@yahoo.com.mx, \\ dhernandez1@dsic.upv.es, miguel.casillas@gmail.com,vic.zamudio@ieee.org
}

\begin{abstract}
Resumen. La tecnología, al igual que las ciencias exactas y la computación juegan un papel muy importante en la vida diaria, así mismo, son de gran ayuda para la resolución de problemas de diferente índole, tales como problemas médicos, tecnológicos, la industria armamentista, escolares, entre otros. En este ocasión, se muestra un enfoque orientado a mejorar la comunicación no verbal de las personas y, a su vez, brindar una alternativa de mejora en la calidad de vida de pacientes con problemas de comunicación verbal. La unión de éstas desencadena en aplicaciones de gran utilidad y brinda alternativas eficaces de comunicación. Por tal motivo, en este trabajo se presenta el desarrollo del proyecto denominado Speechless Talk cuyo principal objetivo consiste en interpretar de manera dicotómica palabras tales como "SI" o "NO" a través de señales neuronales biológicas obtenidas con el uso de EEG (Electroencefalogramas), que posteriormente son clasificadas utilizando Redes Neuronales Artificiales.
\end{abstract}

Palabras clave: Redes neuronales artificiales, EEG (electroencefalograma), MindFlex, dicotomía, interfaces cerebro máquina.

\section{Cognitive Bias and Artificial Neural Networks Applied in a BCI for Classification of Biological Neural Signals to Dichotomous Words "YES-NO" Obtained Through an EEG: Speechless Talk}

Abstract. Technology, like the exact sciences and computers play a very important role in daily life, likewise, they are of great help for the 
resolution of problems of different nature, such as medical, technological problems, the arms industry, school, among others. On this occasion, an approach is shown aimed at improving the non-verbal communication of people and, at the same time, providing an alternative to improve the quality of life of patients with verbal communication problems. The union of these triggers in applications of great utility and offers effective alternatives of communication. For this reason, this paper presents the development of the project called Speechless Talk whose main objective is to interpret in a dichotomous way words such as "YES" or "NO" through biological neural signals obtained with the use of EEG (Electroencephalograms), which are subsequently classified using Artificial Neural Networks.

Keywords: ANN (articial neural network), EEG (electroencephalogram), MindFlex, dichotomous, BCI (brain computer interfaces).

\section{Introducción}

Según el INEGI (Instituto Nacional de Estadística y Geografía), para el 2010 existían un total de 4,527,784 casos documentados con personas que presentan algún tipo de discapacidad en México, de los cuales el $8.93 \%$ (es decir, aproximadamente 401,538 personas [12]), presentan algún tipo de problema para comunicarse de manera oral.

Lo cual trae consigo una gran problemática en varios sectores, partiendo desde el paciente que se encuentra postrado en cama y no puede expresar de manera adecuada sus necesidades o síntomas (dificultando con esto la comunicación paciente-médico), así como la frustración que puede llegar a sentir dicho paciente al no poder expresar de manera adecuada lo que requiere, hasta la persona que necesita realizar sus actividades cotidianas lo mas normal posible y no tiene forma alguna de comunicarse.

Por otra parte, personas que se encuentran en estado vegetativo, mientras conserven la conciencia, podrían tener una alternativa de comunicación con sus familiares, médicos y personal que los atiende.

La tecnología juega un papel muy importante en nuestras actividades cotidianas y cada día es más frecuente acceder a esta, por tal motivo, se considera de suma importancia la implementación de soluciones tecnológicas a problemas comunes y apoyar a mejorar la calidad de vida de las personas.

Un buen ejemplo, es el caso del científico Steven Hawking quien padecía ELA (Esclerosis Lateral Amiotrófica) ${ }^{3}$. Para mejorar la forma de comunicación de Hawking, se creó una versión mejorada de la tecnología Swiftkey, que consiste en la interpretación de frases a través de palabras detectadas por una serie

${ }^{3}$ ELA es una enfermedad degenerativa de tipo neuro-muscular,que se caracteriza por destruir células y neuronas motoras, tanto en extremidades como en la musculatura bulbar [1], de manera paulatina 
de sensores colocados tanto en la mejilla derecha así como en la garganta del usuario, para que cuando este emule hablar, los sensores interpreten las palabras que intentó pronunciar.

Dichas palabras se someten al software Swiftkey el cual intenta colocarlas de la mejor manera posible. Para realizar dicha predicción, el software tiene precargadas todas y cada una de las publicaciones realizadas por Hawking hasta el día de su muerte (14-03-2018), para así encontrar coincidencias en lo que comúnmente es llamado "forma de escribir" y determinar la frase exacta de lo que se emula pronunciar.

Una vez que se cuenta con la interpretación, una computadora colocada en la silla de ruedas del científico se encargaba de leer a través de los altavoces, lo que Hawking ha querido pronunciar.

El nombre de dicho sistema es ACAT (Assistive Context-Aware Toolkit), desarrollado por la empresa INTEL [13]; una de las principales desventajas de este completo sistema es su elevado precio de producción, lo cual lo hace inaccesible para el público en general.

Otro gran avance con el que contamos hoy en día es el proyecto denominado Brain-To-Text [2] desarrollado por la Universidad de Bremen en Alemania, el cual consiste en la traducción de ondas cerebrales a texto, mediante un algoritmo diseñado por ellos mismos.

Para poder realizar dicha prueba, Herff y su equipo de trabajo tuvieron que movilizarse a un hospital en EEUU especializado en pacientes con epilepsia. Los experimentos se consideran un método totalmente invasivo, ya que los electrodos son colocados directamente en el encéfalo para obtener los datos; como resultado se obtuvieron un total de 7 palabras bien clasificadas de diez almacenadas en una base de datos, pero cuando se incrementan las palabras a 100, únicamente 43 de ellas fueron correctamente clasificadas [2]. Al ser un método totalmente invasivo, se considera poco funcional y de difícil acceso al público en general.

El proyecto denominado "habla imaginada" desarrollado por la red ICA (Inteligencia Computacional Aplicada) en el INAOE (Instituto Nacional de Astrofísica, Óptica y Electrónica), consiste en la clasificación de cinco palabras (Izquierda, Derecha, Arriba, Abajo, Seleccionar) [3] obtenidas de la actividad cerebral apoyados en potenciales visuales evocados; dicho proyecto cuenta con un $70 \%$ de clasificación correcta lo que lo hace un aporte bastante confiable, para este caso se utiliza una diadema denominada EMOTIV Epoc que cuenta con 14 canales para la obtención de señal y tiene un costo alto, adicionalmente, se requiere de un entrenamiento especial para poder implementar dicho sistema.

Con el objetivo solucionar los problemas de comunicación oral, se propone la implementación de un sistema de comunicación no verbal que permita a pacientes con comunicación oral limitada o nula, expresar de manera sencilla sus necesidades básicas, así como expresar incomodidades o responder cosas tan básicas como si tiene hambre, le duele algo o tiene alguna incomodidad en el momento, a través de un simple "SI" o "NO".

Por tal motivo, se crea Speechless Talk, que es una aplicación de escritorio que permite a personas con comunicación limitada a nula, expresar palabras 
básicas, tales como: "SI" o "NO", solamente con pensarlo; Apoyados en tecnicas computacionales inteligentes.

\section{Marco referencial}

Para brindar una alternativa de comunicación no verbal y ofrecer una mejora a las técnicas computaciones existentes hasta hoy en día, se presenta el proceso de comunicación a nivel neuronal, que toma en cuenta que necesitamos realizar un prototipo económico y que no requiera de estímulos externos diferentes al de pensar. Dado que en psicología existe un término conocido como "sesgo cognitivo", el cual indica que existe cierta información básica almacenada en algunas neuronas y dicha información está presente casi por inercia (tales como nuestro nombre, nuestra edad, nombre de nuestros padres, lugar donde vivimos, entre otros) también indica que existe información que requiere mayor esfuerzo para acceder a ella o para procesarla por ejemplo la raíz cuadrada de 1326, recordar que comimos ayer por la mañana, o pensar de que color estaba vestido el profesor de matemáticas, etc. [5] Esta información a su vez puede ser causante de algunos errores en la toma de decisiones de la vida diaria.

Bajo este principio y las teorías que indican que a nivel neurológico la comunicación entre neuronas se realiza a través de pulsaciones eléctricas enviadas de neurona a neurona $[6,7,10,11]$, se infiere que cuando nos hacen preguntas básicas tales como: ¿Te llamas Carlos?, ¿Ya comiste?, ¿El cielo es azul?, etc. nuestra respuesta por inercia es un "SI" o "NO". Dicha respuesta no requiere de una gran cantidad de actividad cerebral, por lo cual con la tecnología de electroencefalografía con la que se cuenta hoy en día, es sumamente difícil de localizar la parte exacta donde se procesó, en un universo de mas de 100000 000000 de neuronas según Belmonte [11]. Por otro lado, si la pregunta fuera: ¿Cuál es la raíz cuadrada de 1536?, la respuesta requeriría de un mayor nivel de concentración y mayor activación cerebral [5], lo cual facilita localizar y/o identificar un patrón que indique a nivel cerebral qué se está pensando en algo, aunque se desconozca en que se piensa, la actividad cerebral es más fácil de localizar.

Por tal motivo y con base en este conocimiento, se infiere que si dedicamos especial atención a pensar en repetidas ocasiones ciertas respuestas, la actividad cerebral generada se captaría con mayor facilidad. Considerando lo anterior, se propone crear un sistema denominado Speechless Talk que consiste en la creación de una aplicación que permita a personas con problemas de comunicación expresar palabras dicotómicas tales como "SI" y "NO", utilizando un EEG desarrollado por la empresa NeuroSky y generando mayor actividad cerebral al pensar en repetidas ocasiones en la misma respuesta.

\section{Desarrollo}

Para la creación del sistema Speechless Talk se requirieron conocimientos básicos en electrónica, programación, redes neuronales artificiales y biológicas, 
Sesgo cognitivo y redes neuronales artificiales aplicados en una BCI para clasificación ...

neurociencias y psicología así como de la diadema de EEG Mindwave, diseñada por NeuroSky, arduino y demás herramientas de trabajo, puesto que es requerido crear una interfaz completa, útil, económica y poco invasiva.

Para lograrlo se utilizó "Mindwave", un EEG [8] que consta de una diadema de 1 Electrodo tipo seco [16]), este se coloca en la posición Fp1 según el sistema internacional 10-20 EEG para la colocación de electrodos o lóbulo frontal; de forma más precisa, en la parte superior del ojo izquierdo, esto debido a que aquí se encuentra la corteza frontomedial (CFM) que es la que se encarga de los procesos de inhibición en la detección y solución de conflictos [14], así como de la regulación y el esfuerzo empleado en la concentración [14]; esto para recibir pulsaciones eléctricas provenientes de la actividad cerebral generada al concentrarnos y meditar; adicionalmente, se cuenta con otros dos electrodos que se colocan en los lóbulos de la oreja, con la finalidad de obtener GND y asegurar la correcta postura de la diadema [8].

Dicha diadema incluye la tecnología ThinkGear [8], dentro de la cual se procesa la señal obtenida por los sensores, además se ejecuta el algoritmo eSense (Algoritmo diseñado por NeuroSky para obtener en una escala del 1 al 100 [9], los niveles de concentración y meditación, en tiempo real así como brindar las señales de las diferentes frecuencias) de la diadema podemos extraer 11 datos, que son: Nivel de Señal, Niveles de Atención, Niveles de Meditación, Delta (1-3Hz), Theta (4-7Hz), Low Alpha (8-9Hz), High Alpha (10-12Hz), Low Beta (13-17Hz), High Beta $(18-30 \mathrm{~Hz})$, Low Gamma $(31-40 \mathrm{~Hz})$, High Gamma [4]; cuyo comportamiento similar al mostrado en la Fig. 1, las cuales son preprocesados en su paso por el algoritmo eSense para discriminar la mayor cantidad posible de ruidos [4].

La diadema recibe a través de los sensores conectados, los valores de las diferentes frecuencias presentadas en ese momento y posteriormente son procesadas con el algoritmo eSense incluido en el chip de la diadema, con tecnología Thinkgear [9].

Prototipo Hardware. Para cumplir el objetivo de clasificar correctamente las palabras "SI" y "NO" a través de la actividad cerebral, es necesario acceder a los datos que se procesan en el chip de la diadema, de tal manera que para desarrollar un prototipo se requiere contar con todos los materiales tales como Diadema EEG NeuroSky (podría ser MindFlex o MindWave), Arduino uno, Matlab, Software Arduino, Arduino Brain Library [15]. En la Figura 2 se muestran los componentes de la diadema, y en la Figura 3 se muestra el circuito de conexión de la diadema con el arduino, así como los puertos del chip ThinkGear para realizar la conexión entre la diadema y el módulo de arduino.

Para establecer la comunicación entre el EEG y la computadora, es requerido conectar por medio de USB el modulo arduino, seguido de una librería desarrollada por Erik Mika [4] en el software de arduino para la recepción de los datos del EEG [15]; para esto, se debe asegurar que la comunicación entre ambos dispositivos se realice con éxito.

Para identificar las señales del EEG y para clasificarlas se emplea una red neuronal. Se utiliza la función patternnet de Matlab; dicha función crea una 

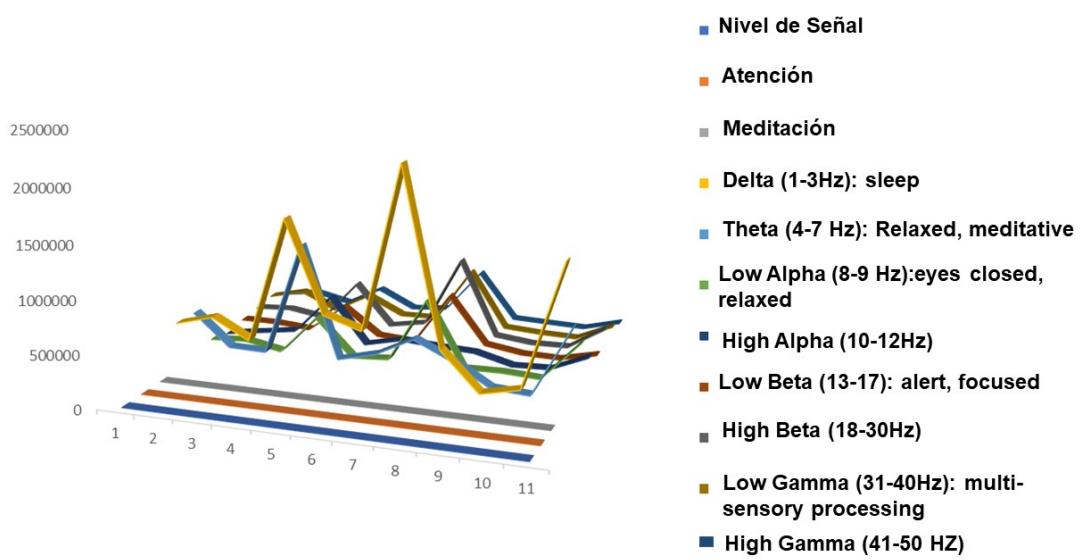

Fig. 1. Diagrama de comportamiento de la señal en las diferentes frecuencias, en 10 segundos.

red neuronal artificial de tipo feedforward cuya función objetivo consiste en diferenciar un "SI" de un "NO"; la estructura de la red la podemos observar en la Fig. 4.

La metodología para diferenciar entre "SI" y "NO", se define de la siguiente manera:

Etapa de entrenamiento:

- Seleccionar un espacio adecuado para pruebas, se recomienda que sea un lugar cerrado o con pocos distractores.

- Explicar al usuario las instrucciones y pedirle que las cumpla al pie de la letra.

- Iniciar la aplicación Speechless Talk en una computadora (desarrollada en Matlab).

- Conexión de diadema Mindwave modificada en el usuario.

- Realizar la conexión del modulo arduino de la diadema y la computadora.

- La aplicación debe comenzar a mostrar una cantidad de 60 preguntas al usuario, las cuales podrán ser escuchadas o leídas por el usuario (según su elección).

Las preguntas deben ser divididas en bloques de 30, cuyas respuestas sean obvias para que la respuesta forzosamente sea un "SI" y otras 30 preguntas cuyas respuestas sean forzosamente un "NO".

- Una vez que el usuario lee y/o escucha la pregunta, debe comenzar a pensar en la respuesta en repetidas ocasiones, hasta que aparezca la siguiente pregunta (aproximadamente 10 segundos). Esto con la finalidad de generar a través de la actividad cerebral una respuesta "SI" o bien una respuesta "NO" y diferenciar ambas con mayor facilidad. 


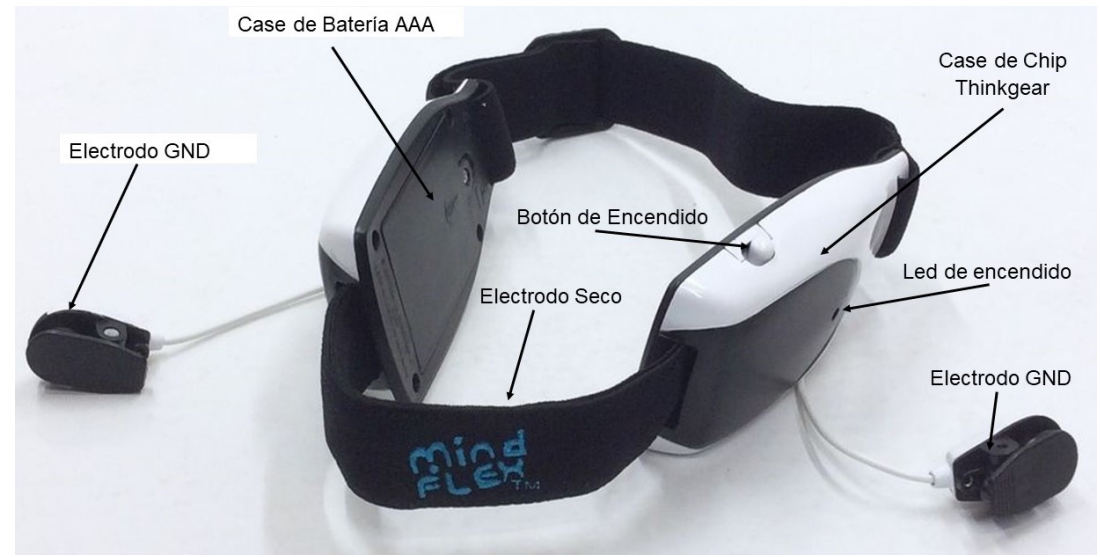

Fig. 2. Diagrama de los componentes de la Diadema Mindflex.

- A través de la diadema EEG, se obtiene la información de la actividad cerebral generada en los 10 segundos que tiene el usuario para responder cada pregunta; esta se obtiene en forma de una señal eléctrica que se introduce al arduino y posteriormente a la computadora, esto se hace continuamente durante las 60 preguntas (aproximadamente 15 minutos, por usuario). La nueva matriz de datos obtenida al finalizar las 60 preguntas será de dimensiones $600^{*} 11$, haciendo referencia a las 600 lecturas obtenidas por el EEG y las 11 características que proporciona el EEG.

- Con la nueva matriz obtenida, entrenar nuestra red neuronal.

Para la etapa de clasificación:

- Realizar una pregunta adicional al usuario.

- Clasificar dentro de la red el resultado a la pregunta realizada.

- Imprimir en pantalla el resultado obtenido.

- Repetir los últimos 3 pasos, 9 ocasiones mas, haciendo un total de 10 preguntas realizadas al usuario; por prueba.

- Comparar la respuesta real esperada con la respuesta de clasificación de la red.

\section{Pruebas y resultados}

Las pruebas se realizan en el Laboratorio de Ambientes Inteligentes del Instituto Tecnológico de León, esto debido a que presenta el ambiente requerido, puesto que no existe demasiado ruido ni mucho trafico peatonal. No fue necesario realizar modificación alguna al laboratorio, tampoco se impidió el acceso a personas mientras se realizaban las pruebas, únicamente se les solicito hablar en voz baja para evitar distraer al usuario; el motivo por el que se realiza una 


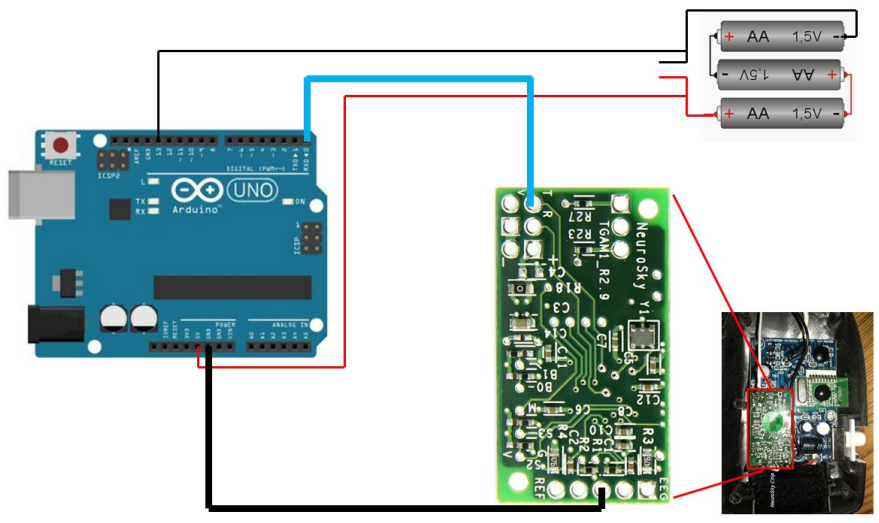

Fig. 3. Circuito Eléctrico de Conexión diadema-Arduino-Alimentación de energía.

Pattern Recognition Neural Network (view)
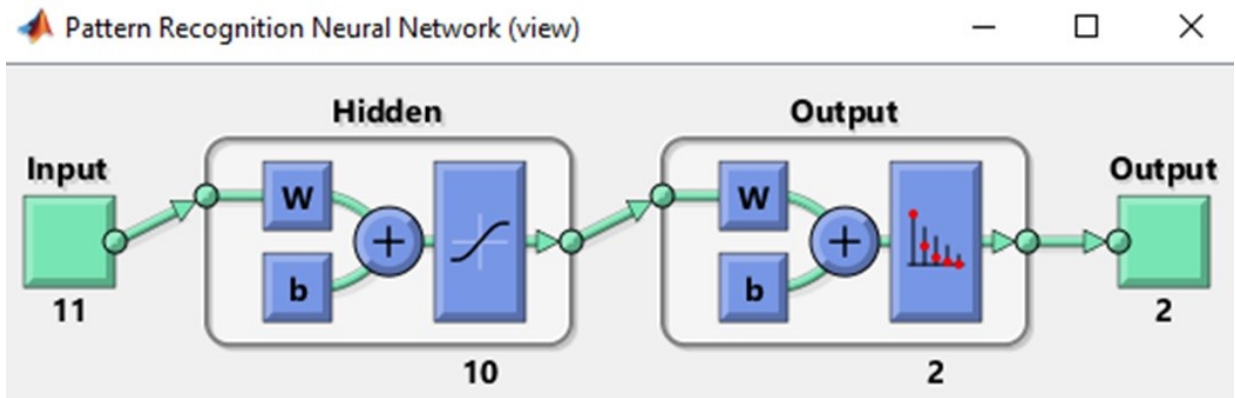

Fig. 4. Estructura de la Red Neuronal.

gran cantidad de preguntas para entrenamiento (60) es precisamente para que sí en algún momento existen factores externos que distraigan al usuario, esto no afecte en su resultado final. Se realizaron pruebas con sujetos de diferente nivel educativo, oficio, género y edad, esto para cubrir distintas características en la población, así como en diferentes horarios y bajo diferentes situaciones, tales como: después de comer, por la mañana, al anochecer, antes de comer, en situaciones de estrés.

Para realizar las pruebas, se indicaron las instrucciones a los usuarios: desde concentrarse en las respuestas, pensar su respuesta en repetidas ocasiones hasta que se le dicte la siguiente pregunta, no poner atención a lo que ocurra en su entorno, solamente pensar y no hablar las respuestas, entre otras, posteriormente, se ejecutó la aplicación y el usuario debe concentrarse solamente en responder con el pensamiento.

Los conjuntos de datos obtenidos por cada sujeto prueba, dentro de la etapa de entrenamiento se utilizaron para entregar y calibrar la red neuronal, la cual 


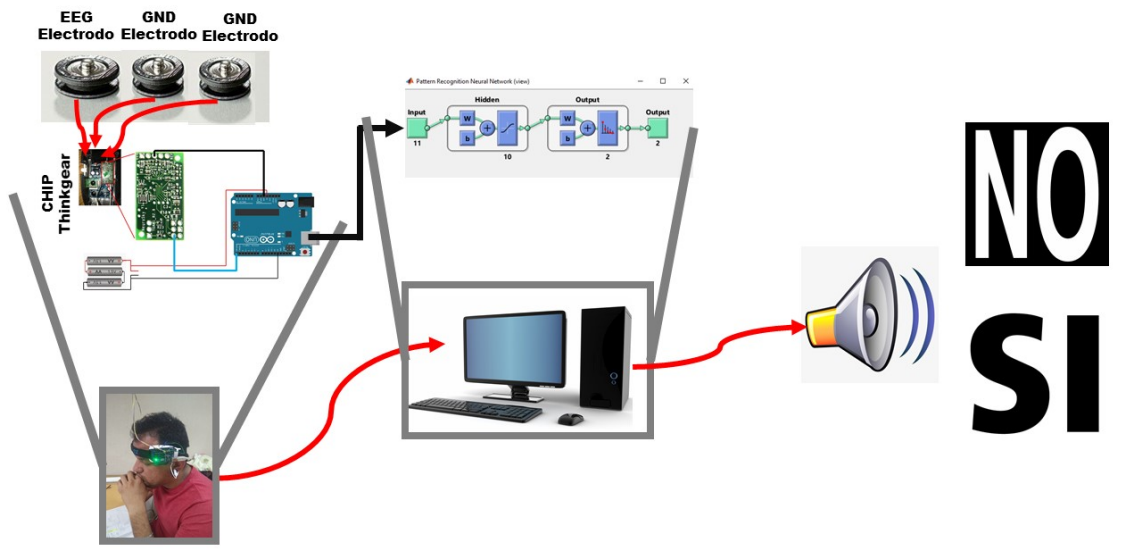

Fig. 5. Diagrama de Speechless Talk.

demostró comportarse como se presenta en la Fig. 6.

De dicha base de datos de señales se hizo una partición de $70 \%$ para entrenamiento de la red, $15 \%$ para validación y $15 \%$ restante se utiliza para pruebas, obteniendo un porcentaje de clasificación del $94.3 \%$ lo cual puede ser corroborado en la Fig. 6. A continuación, en la Fig. 7, vemos el comportamiento de las curvas ROC (receiver operating characteristic curve) utilizadas para la representación de falsos positivos frente a la razón de verdaderos positivos.

Como resultado de la experimentación, se obtiene una aplicación de escritorio que se conecta por un puerto serial a un EEG colocado en el cráneo del usuario, dicha aplicación incluye una red neuronal de tipo feedforward que se encarga de clasificar los pensamientos del usuario y obtener una respuesta a las preguntas en cuestión; la primer versión solamente clasifica las palabras "SI" y "NO"; dicha red, así como toda la programación de la aplicación, se encuentra desarrollada en Matlab.

El sistema se encarga de leer y mostrar a los usuarios una serie de preguntas a las cuales se debe responder solamente pensando, después de una serie de 60 preguntas, viene una serie adicional de preguntas con base a lo que se desea saber, cuyas únicas respuestas deben ser "SI" o "NO" (en esta parte es donde se predice o se realiza la comunicación en tiempo real con el usuario). Para la parte de prueba, se sometieron al experimento 15 personas y se observó el siguiente comportamiento en los resultados de clasificación entre las respuestas esperadas y las respuestas que clasificó la el sistema Speechless Talk.

Cabe mencionar que se tienen 106 preguntas correctamente clasificadas de un total 150 , lo que hace un $71.33 \%$ de clasificación correcta. 

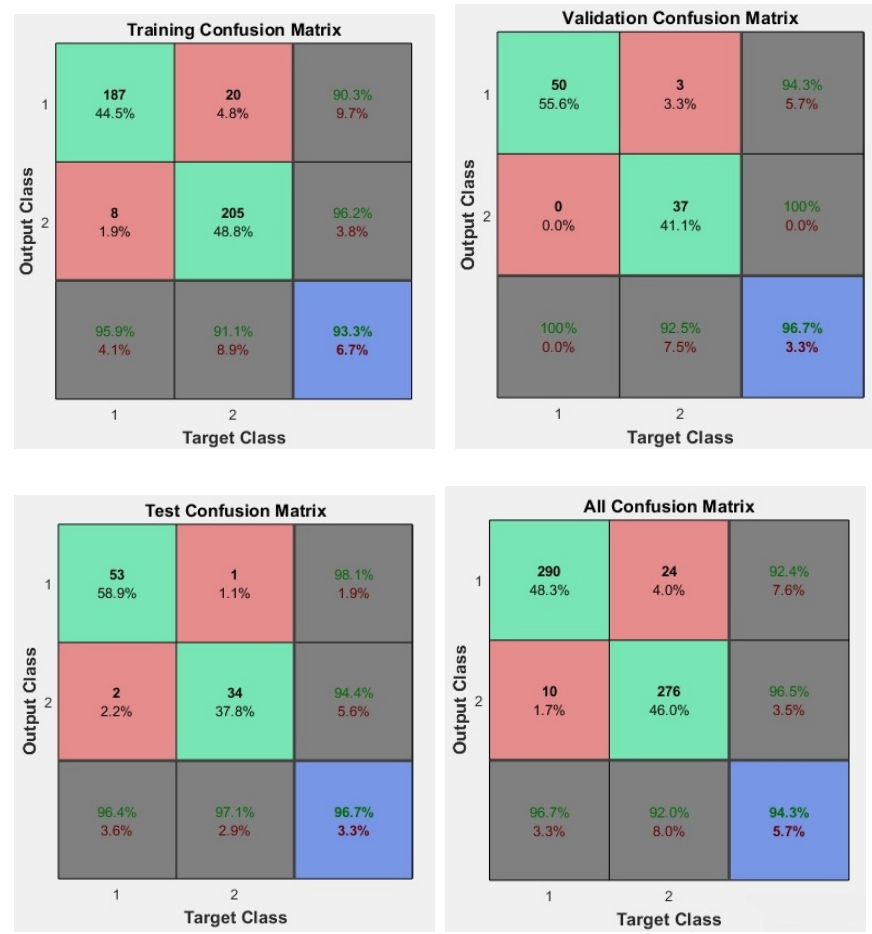

Fig. 6. Matriz de confusión.

\section{Conclusiones}

El trabajar con señales que provienen de EEG, es un tema complejo, además, existe una serie de factores que afectan los resultados esperados y se deben de tomar en cuenta, como la alimentación, el estado anímico, la hora del día, estado de salud, así como el entorno que rodea a los usuarios, ya que si bien, por la mañana, probablemente el usuario se encuentre en un estado más activo, presente diferencias a la hora de concentrarse, así mismo, dependiendo lo que el usuario haya comido puede llegar a acelerar la actividad neuronal, lo cual generaría interferencia para pruebas futuras, en un conjunto de datos ya entrenado.

$\mathrm{Al}$ realizar las 60 preguntas de entrenamiento al usuario e inmediatamente presentar las 10 preguntas de prueba, los resultados presentaban cierto sesgo hacia las últimas preguntas realizadas, esto debido a que los niveles de somnolencia o cansancio juegan un papel muy importante al momento de clasificar y es considerado como factor decisivo en la prueba en tiempo real, pues el paciente se encuentra en el mismo estado que cuando presentó las ultimas 30 preguntas; por consiguiente las clasificación de la red neuronal artificial se inclina por la respuesta pensaba en las ultimas 30 preguntas de entrenamiento. 

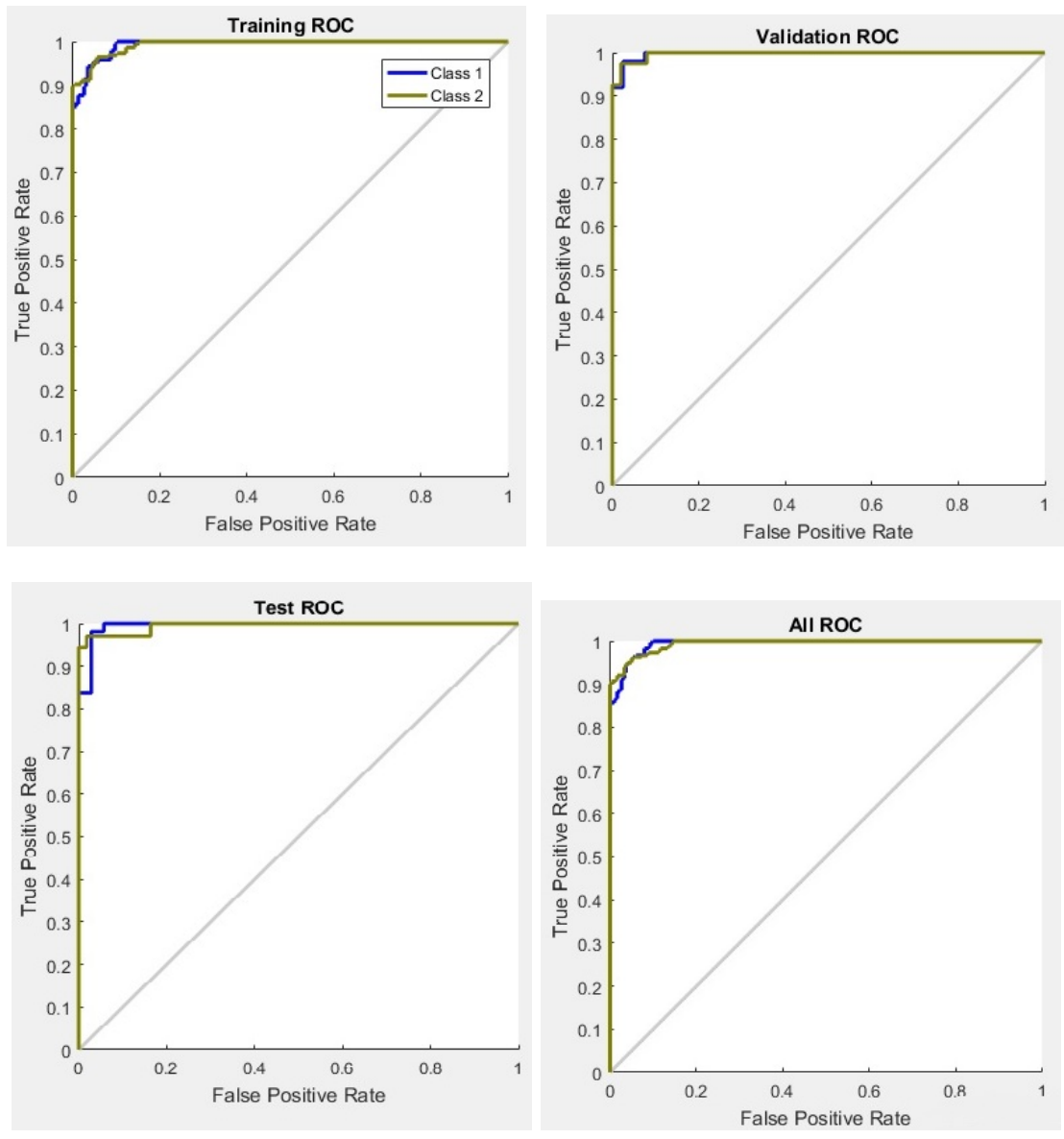

Fig. 7. Análisis de curvas ROC (receiver operating characteristic curve).

Otro factor a considerar para la mejora de resultados es la disponibilidad del usuario, debido a que después de algunos minutos los usuarios para prueba llegan a sentir aburrimiento o cansancio o no prestan la atención necesaria a la prueba, generando con esto, resultados erróneos. Para el correcto funcionamiento y mejorar la cantidad de respuestas correctas, se solicita a los usuarios pongan todo el empeño necesario en concentrase.

Se analizaron diferentes técnicas de inteligencia computacional para la correcta clasificación de señales neuronales biológicas, así como métodos de tratamiento de señal, optando por emplear una red neuronal artificial de tipo feedforward; se diseñó una BCI amigable con el usuario para obtener las pulsaciones eléctricas de los usuarios y enviarlas a una computadora para ser analizadas y clasificadas en la red neuronal programada en Matlab, dando como resultado un $71.33 \%$ palabras dicotómicas "SI" y "NO" obtenidas al pensar, las cuáles son respuestas 
Tabla 1: Comparativa de respuesta paciente vs respuesta Speechless Talk. PD: 0 indica una respuesta NO y 1 Indica una respuesta SI.

\begin{tabular}{|c|c|c|c|}
\hline & \multicolumn{3}{|c|}{ Speechless Talk } \\
\hline & Respuestas Paciente & Respuesta Speechless Talk & Porcentaje de clasificación \\
\hline Sujeto 1 & $\begin{array}{lllllllllll}1 & 0 & 1 & 1 & 0 & 0 & 1 & 0 & 1 & 1\end{array}$ & $\begin{array}{lllllllllll}1 & 0 & 1 & 1 & 0 & 1 & 1 & 0 & 1 & 1\end{array}$ & $90 \%$ \\
\hline Sujeto 2 & 10011001011 & 11101000101011 & $80 \%$ \\
\hline Sujeto 3 & $\begin{array}{lllllllllll}0 & 1 & 0 & 1 & 0 & 1 & 0 & 0 & 0 & 0\end{array}$ & $0 \begin{array}{llllllllll}0 & 1 & 0 & 0 & 0 & 1 & 1 & 0 & 0 & 0\end{array}$ & $80 \%$ \\
\hline Sujeto 4 & $\begin{array}{lllllllllll} & 0 & 0 & 1 & 0 & 1 & 0 & 0 & 0 & 0\end{array}$ & $\begin{array}{lllllllllll}0 & 0 & 0 & 1 & 0 & 0 & 0 & 1 & 0 & 0\end{array}$ & $80 \%$ \\
\hline Sujeto 5 & 0000010001000 & 0000000000000 & $80 \%$ \\
\hline Sujeto 6 & $\begin{array}{|llllllllll|}0 & 0 & 0 & 0 & 1 & 0 & 0 & 1 & 0 & 0 \\
\end{array}$ & $0 \begin{array}{llllllllll} & 0 & 0 & 0 & 0 & 0 & 0 & 1 & 1 & 0\end{array}$ & $80 \%$ \\
\hline \begin{tabular}{|l|} 
Sujeto 7 \\
\end{tabular} & $\begin{array}{llllllllll} & 0 & 0 & 1 & 0 & 0 & 0 & 0 & 0 & 1\end{array}$ & $\begin{array}{llllllllll}1 & 0 & 1 & 1 & 0 & 1 & 0 & 0 & 0 & 1\end{array}$ & $70 \%$ \\
\hline Sujeto 8 & $\begin{array}{llllllllll}1 & 0 & 1 & 1 & 1 & 0 & 0 & 1 & 1\end{array}$ & $\begin{array}{lllllllllll}0 & 0 & 1 & 1 & 1 & 1 & 0 & 0 & 1 & 1\end{array}$ & $70 \%$ \\
\hline Sujeto 9 & $\begin{array}{lllllllllll}0 & 1 & 0 & 0 & 1 & 0 & 0 & 0 & 0 & 1\end{array}$ & $0 \begin{array}{llllllllll}0 & 1 & 0 & 0 & 1 & 1 & 0 & 0 & 1 & 0\end{array}$ & $70 \%$ \\
\hline Sujeto 10 & 111100010000 & 111010001011 & $70 \%$ \\
\hline Sujeto 11 & $\begin{array}{llllllllllll}0 & 1 & 0 & 1 & 0 & 1 & 1 & 0 & 0 & 0\end{array}$ & $\begin{array}{lllllllllll} & 0 & 1 & 1 & 1 & 1 & 1 & 0 & 0 & 0\end{array}$ & $70 \%$ \\
\hline Sujeto 12 & 000000111111111 & 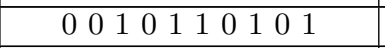 & $70 \%$ \\
\hline Sujeto 13 & $\begin{array}{llllllllll}10 & 1 & 1 & 1 & 0 & 1 & 1 & 0 & 1\end{array}$ & $\begin{array}{lllllllllll} & 0 & 0 & 1 & 1 & 0 & 1 & 1 & 0 & 0\end{array}$ & $70 \%$ \\
\hline Sujeto 14 & 1001011100 & 111110001011 & $60 \%$ \\
\hline Sujeto 15 & $\begin{array}{lllllllllll}0 & 1 & 1 & 1 & 1 & 1 & 1 & 0 & 0 & 1\end{array}$ & $\begin{array}{lllllllllll}0 & 0 & 0 & 0 & 0 & 0 & 0 & 0 & 0 & 0\end{array}$ & $30 \%$ \\
\hline
\end{tabular}

a una serie de preguntas realizadas a 15 usuarios diferentes, en tiempo real.

Speechless Talk es una aplicación pensada en apoyar a todas aquellas personas que cuentan con algún problema de comunicación verbal, la cual a través de una interfaz BCI obtiene señales obtenidas al pensar y estimular los niveles de concentración para posteriormente a través de técnicas computacionales inteligentes procesar las señales obtenidas y clasificar lo mejor posible las señales que conforman el pensamiento dicotómico para las palabras "SI" y "NO".

\section{Trabajo futuro}

Después del trabajo realizado, se sugieren las siguiente mejoras para las posteriores versiones:

- Implementar filtros de tratamiento de señal.

- Experimentar con diferentes técnicas de clasificación para mejorar resultados.

- Incrementar la cantidad de palabras a clasificar.

- Realizar un prototipo hardware portátil para brindarle mayor movilidad e independencia al paciente.

- Implementar Speechless Talk en aplicaciones médicas.

\section{Referencias}

1. Zapata-Zapata, C.H., Franco-Dager, E., Solano-Atehortua, J.M., AhuncaVelasquez, L.F.: Amyotrophic lateral sclerosis: update/Esclerosis lateral amio- 
Sesgo cognitivo y redes neuronales artificiales aplicados en una BCI para clasificación ...

trofica: actualizacion/La esclerose lateral amiotrofica, Universidad de Antioquia, Facultad de Medicina (2016)

2. Herff, C., Heger, D., Pesters, A., Telaar, D.: Brain-to-text : decoding spoken phrases from phone representations in the brain. Frontier in Neuroscience, 2(5), pp. 1-11 (2015)

3. Torres-García, A.A., Reyes-García, C.A., Villaseñor-Pineda, L., García-Aguilar, G.: Implementing a fuzzy inference system in a multi-objective EEG channel selection model for imagined speech classification. El Sevier, pp. 1-12 (2016)

4. Brain-Hack, M.E.: http://www.frontiernerds.com/brain-hack (2018)

5. Kohan, N.C.: http://www.redalyc.org/articulo.oa?id=299023503010 (2011)

6. Gupta, K.C.: Neural Network Structures. Neural Networks for RF and Microwave Design. pp. 61-103 (2000)

7. Bear M., Connors, B., Paradiso, M.: Neurociencia La Exploración del Cerebro. The Plant Journal, 41 (2005)

8. NeuroSky: http://developer.neurosky.com/docs/doku.php?id=what_is_ thinkgear (2018)

9. NeuroSky: http://developer.neurosky.com/docs/doku.php?id=esenses_tm\& s [] =esense (2018)

10. Purves, D., Augustine, G., Fitzpatrick, D., Hall, W., Lamatia, A., Mcnamara, J.W.M.: Neurociencia. 918 (2008)

11. Belmonte, C.: La exploración del cerebro : Una aventura de futuro (2012)

12. INEGI: http://www.beta.inegi.org.mx/temas/discapacidad/ (2017)

13. INTEL: https://01.org/acat/documentation-list (2017)

14. Flores-Lázaro, J. C.: Neuropsicología de Lóbulos Frontales, Funciones Ejecutivas y Conducta Humana. Revista Neuropsicología, Neuropsiquiatría Y Neurociencias, 8(1), pp. 47-58 (2008)

15. NeuroSky: http://developer.neurosky.com/docs/doku.php?id=projects (2018)

16. Vea-Murgía, V.P.: Investigación del Funcionamiento de Electrodos Secos y Gorro de Diseño Propio contra Gorro Comercial con Electrodos Húmedos Aplicando Filtros CSP a Tareas de Movimiento. 34 (2011) 